\title{
Generated meaningfulness of single letters as a function of position in CVC trigrams ${ }^{1}$
}

CALVIN F. NODINE AND JAMES V. HARDT CARNEGIE-MELLON UNIVERSITY

A method of computing generated meaningfulness values (GM) of single letters as a function of their position (initial, middle, terminal) within CVC trigrams is presented. $G M$ is based on Archer (1960) meaningfulness values ( $M$ ) for all $2100 \mathrm{CVC}$ trigrams. GM represents the contribution to $M$ of a given letter in a given position after all "residual" effects have been averaged out. The results show that differences in $M$ are associated with different letters in a given position. High correlations between $G M$ and letter frequency lend validity to the concept of $M$.

The purpose of this paper is to examine the relationship between Archer (1960) meaningfulness (M) of trigram units and generated meaningfulness (GM) of specific individual letters as a function of their position within these trigram units. The question of concern is whether specific letters in initial, middle and terminal positions of CVC trigrams are associated with differences in M. Underwood \& Schulz (1960) have shown that frequency of occurrence of letter sequences in words is related to the strength of letter associations. Method

To obtain GM for a specific letter in a given position, the sum of $M$ values for all trigrams for a given position was obtained. The sum was divided by the number of contributing trigrams, and the resulting value is GM. For example, to obtain GM for the initial consonant " $B$ " the $M$ values for all trigrams beginning with " $B$ " were summed and divided by the total number of trigrams with an initial "B." The operations for obtaining GM for " $B$ " is more explicitly shown in the formula: GM (Binitial) $=$

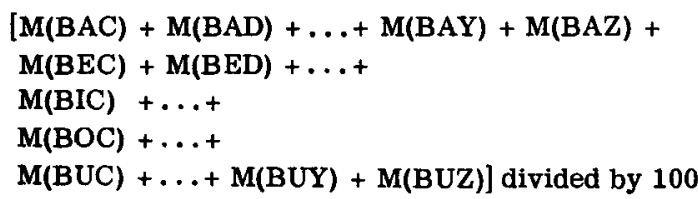

From the example, it is clear that GM for a specific letter in a given position (e.g., "B') reflects the residual $M$ after all other effects have been averaged out. Because all consonants appear in both initial and terminal positions in CVC trigrams, initial and terminal consonants are each composed of 100 "bigram" combinations (5 vowels $\times 20$ consonants). For a given vowel, 420 "bigram" combinations contributed to the sum (21 initial consonants $\times 20$ terminal consonants). The constraints on the form of the CVC's which resulted in the initial consonant, middle vowel, and terminal consonant sums of 100,420 and 100 respectively were: (1) Only "A", "E", "I", "O", and "U" are vowels
(' $Y$ "' is a consonant); (2) no duplication of letters in initial and terminal positions (e.g., BAB, CAC etc.) Results

The GM values and standard deviations for letters as a function of position are shown in Table 1. As one might suspect, initial "X", "Q", "Z", "Y", and "V"' are of low GM indicating that trigrams beginning with these letters are generally of low M. Initial "H", "S", " $R$ ", and " $D$ " have high GM values indicating that trigrams beginning with these letters are generally high in $M$. The implications are similar for terminal " $J$ ", "Q", and "H" which are of low GM and " $\mathrm{N}$ ", "T", and " $L$ " which are of high GM. "A" in the vowel position has high GM whereas "U'" has low GM. The relationship between GM and $M$ has documented what verballearning researchers have "known" for years, namely, that letter-position combinations influence $\mathrm{M}$ of $\mathrm{CVC}$ trigram units.

What is the relationship between GM and the frequency of a letter in a given position? Mayzner \&

Table 1. Means and Standard Deviations of Generated Meaningfulness (GM) Values for Single Letters Based on Archer (1960) Meaningfulness Values for all 2100 CVC Trigrams.

\begin{tabular}{|c|c|c|c|c|c|c|}
\hline \multirow[b]{2}{*}{ Letter } & \multicolumn{2}{|c|}{ Initial Consonant } & \multicolumn{2}{|c|}{ Middle Vowel } & \multicolumn{2}{|c|}{ Terminal Consonant } \\
\hline & Mean GM & S D & Mean GM & S D & Mean GM & S D \\
\hline $\mathbf{A}$ & & & 67.38 & 29.86 & & \\
\hline B & 71.41 & 27.64 & & & 58.80 & 31.73 \\
\hline C & 65.26 & 28.15 & & & 64.08 & 29.67 \\
\hline D & 73.09 & 24.17 & & & 69.84 & 29.45 \\
\hline $\mathrm{E}$ & & & 56.33 & 29.98 & & \\
\hline F & 63.30 & 27.91 & & & 48.55 & 28.40 \\
\hline G & 56.35 & 31.13 & & & 67.63 & 32.52 \\
\hline $\mathrm{H}$ & 74.42 & 25.24 & & & 35.53 & 19.91 \\
\hline 1 & & & 56.10 & 31.62 & & \\
\hline $\mathbf{J}$ & 61.69 & 28.61 & & & 17.60 & 12.70 \\
\hline K & 58.96 & 27.74 & & & 60.21 & 25.65 \\
\hline $\mathrm{L}$ & 71.82 & 24.37 & & & 74.12 & 25.19 \\
\hline $\mathbf{M}$ & 67.64 & 28.12 & & & 71.57 & 26.22 \\
\hline $\mathrm{N}$ & 63.41 & 26.49 & & & 79.09 & 26.74 \\
\hline $\mathrm{o}$ & & & 60.72 & 30.23 & & \\
\hline P & 69.99 & 26.16 & & & 68.17 & 28.60 \\
\hline$Q$ & 25.24 & 13.57 & & & 30.70 & 16.26 \\
\hline $\mathbf{R}$ & 73.76 & 25.78 & & & 71.32 & 23.92 \\
\hline $\mathrm{S}$ & 74.50 & 25.18 & & & 66.71 & 25.07 \\
\hline $\mathrm{T}$ & 66.32 & 28.36 & & & 77.67 & 27.78 \\
\hline $\mathrm{U}$ & & & 51.35 & 30.90 & & \\
\hline V & 45.11 & 26.60 & & & 47.64 & 27.44 \\
\hline W & 61.99 & 27.44 & & & 54.76 & 32.98 \\
\hline $\mathrm{X}$ & 9.63 & 6.19 & & & 55.30 & 30.02 \\
\hline $\mathrm{Y}$ & 40.26 & 28.65 & & & 54.34 & 31.33 \\
\hline Z & 31.75 & 18.96 & & & 52.27 & 24.22 \\
\hline
\end{tabular}


Table 2. Rank Correlations (Rho) Between GM and Letter Frequency

Initial-Letter GM Terminal-Letter GM

\begin{tabular}{lll}
\hline Total-Letter Frequency ${ }^{1}$ & $.76^{*}$ & $.61^{*}$ \\
Initial-Letter Frequency $^{2}$ & .10 & - \\
Terminal-Letter Frequency $^{2}$ & - & .47 \\
\hline
\end{tabular}

${ }_{1}^{1}$ From Mazyner \& Tresselt (1965) for words of length 3.7.

${ }^{2}$ For words of length 3 only.

Tresselt (1965) have provided tables of single-letter frequency counts as a function of letter position. Correlations between GM and letter frequency are shown in Table 2. Both initial and terminal GM correlate highly with total-letter frequency. It is also clear from Table 2 that the relationship between initial-letter GM and initial-letter frequency of words of length 3 is low; however, this correlation increases from .10 to .71 $(p<.05)$ when initial-letter frequency of all word lengths $(3-7)$ are correlated with GM. The fact that the rho for initial letters is low between GM and three-letter words, and increases when all word lengths are included, lends validity to the concept of $M$ since $M$ presumably reflects number of associations regardless of word length. Differences in the stability of the distributions for different word lengths may also be reflected in these correlations.
The same relationships hold for terminal letters. The rho between GM and terminal letters for all word lengths increases from .47 to .66 ( $\mathrm{p}<.05)$. These correlations suggest that initial letters are more important determiners of $M$ than terminal letters which is consistent with Goss \& Nodine's (1965) suggestion that M may reflect availability of representational responses. Furthermore, although initial letters may be important determiners of $\mathrm{M}$ with nonsense materials, it does not seem to be the case with materials containing semantic content (e.g., Clark, in press).

\section{References}

ARCHER, E. J. A re-evaluation of the meaningfulness of all possible CVC trigrams. Psychol. Monogr., 1960, 74, Whole No. 497.

CLARK, H. H. On the use and meaning of prepositions. J. verbal Learn. verbal Behav., in press.

GOSS, A. E., \& NODINE, C. F. Paired-associates learning. New York Academic Press, 1965.

MAYZNER, M. S., \& TRESSELT, M. E. Tables of single-letter and diagram frequency counts for various word-length and letter-position combinations. Psychon. Monogr. Suppl, 1965, 1, No. 2.

UNDERWOOD, B. J., \& SCHULZ, R. W. Meaningfulness and verbal learning. Chicago: Lippincott, 1960.

Note

1. This research was supported by Grant MH 1197402 from the National Institute of Mental Health, U. S. Public Health Service.

Comment on Serial Isolation by Shock by Louis G. Lippman, Western Washington State College

and Marcia Z. Lippman, The U niversity of British Columbia

In a recent issue of this journal, Raskin, Hattle, \& Rubel (1967) reported the results of a serial isolation experiment in which the experimental group (SI) received an electric shock paired with the seventh item of a 12-item CVC list. One control group (C) received no shock, while the other control group (CS) received shock during the intertrial interval. Contrary to the typical results of isolation experiments-i.e., no facilitation of total list acquisition from isolation-these investigators found total list learning to be significantly faster in Group SI than in Group C or CS. Raskin et al attributed their results to increased attention, produced by the evoking of an orienting reflex by electric shock in contiguity with the seventh serial item (Group SI). It is suggested here that there may be additional factors which may not only help account for the enhanced total list acquisition in Group SI, but which may also put this study into relation with other isolation studies in the serial learning literature. One of these factors has its source in differences between isolation by shock and the conventional method of isolation by color-and hypothetical functional differences which are outcomes of these methodological differences in item Isolation. The other factor involves S's set and style of learning, as modified by the presence of electric shock.
With the traditional method of isolating by color, the isolated and nonisolated lists are identical except that a distinctive feature (color) is embedded into the elements (letters) of the to-be-learned isolated term. An isolate of this type may be learned more rapidly than its nonisolated counterpart in a control list, but this advantage is at the "expense" of other items in the series (Wallace, 1965). More specifically, Newman \& Saltz (1958) have suggested that isolation of an item in the middle of a serial list fails to enhance total list learning because isolation produces accelerated learning of the isolated term as a response. As a response, the isolate tends to be emitted frequently at inappropriate positions within the series and thus precludes correct anticipation of other items on the list, thereby interfering directly with total list performance.

Isolating by shock (qua Raskin et al) differs from 1solating by color in two major ways: (1) The isolating stimulus is not spatially proximal to (or embedded into) the to-be-learned item as is the color stimulus; and, perhaps accentuating the absence of spatial proximity, (2) the shock stimulus is in a different sensory modality from the visually presented list item. It is suggested that because of the externality of the shock stimulus to the seventh Item, the item was not emitted as often as an intralist 